\author{
WŁODZISŁAW DuCH \\ WIESEAW NOWAK \\ JAROSŁAW MELLER \\ Grzegorz OsIŃsKI \\ KRzyszTof Dobosz \\ DARIUSZ MIKOŁAJEWSKI \\ Grzegorz M. WóJCIK
}

\title{
COMPUTATIONAL APPROACH \\ TO UNDERSTANDING \\ AUTISM SPECTRUM DISORDERS
}

\begin{abstract}
Every year the prevalence of Autism Spectrum of Disorders (ASD) is rising. Is there a unifying mechanism of various $A S D$ cases at the genetic, molecular, cellular or systems level? The hypothesis advanced in this paper is focused on neural dysfunctions that lead to problems with attention in autistic people. Simulations of attractor neural networks performing cognitive functions help to assess system long-term neurodynamics. The Fuzzy Symbolic Dynamics (FSD) technique is used for the visualization of attractors in the semantic layer of the neural model of reading. Large-scale simulations of brain structures characterized by a high order of complexity requires enormous computational power, especially if biologically motivated neuron models are used to investigate the influence of cellular structure dysfunctions on the network dynamics. Such simulations have to be implemented on computer clusters in a grid-based architectures.
\end{abstract}




\section{Introduction}

In 1943 Leo Kanner defined autism as "extreme aloneness from the beginning of life and an anxiously obsessive desire for the preservation of sameness". He noticed that autistic children "have come into the world with the innate inability to form the usual, biologically provided affective contact with people, just as other children come into the world with innate physical or intellectual handicaps".

The causes of autism seemed to be quite mysterious and initially psychodynamic theories gained popularity, blaming bad parents, "refrigerator cold" mothers and proposing other psychological explanations. While ASD symptoms may be quite diverse a well-documented common deficit is the lack of the theory of mind, inability to see other people as having their own internal points of views.

In the modern view autism is perceived as a developmental and behavioral syndrome, with multiple etiologies, including genetic mutations, metabolic, and immune system deregulation leading to various impairments in neural connectivity. However, as in many areas of neuroscience, we are "data rich and theory poor" [28], lacking good models that would help to understand what is wrong at the fundamental level. In this paper one of possible mechanisms of ASD is linked to attention deficits, simulated using a neural network model with attractor analysis.

\section{Characteristics of Autism}

\subsection{Autism Spectrum of Disorders}

The prevalence of ASD is probably around 1\%, and recent estimation of undiagnosed cases increases it to $1.5 \%$ [2]. About one third of parents of children with ASD noticed a problem before their childs first birthday, and $80 \%$ saw problems by 24 months. The median age of earliest ASD diagnosis is between 4.5 and 5.5 years. ASD incidence strongly depends on sex, about four times more boys suffer from autism than girls. Opinions still differ whether there is an epidemic of autism. ASD occurs in all racial, ethnic, and socioeconomic groups and has huge social and financial costs. Although this disease is typically thought of as a childhood disorder, their costs can be felt well into adulthood. Quantitative estimates made in the Unites States in 2007 put the lifetime cost of care for an autistic individual to be around 3.2 million USD.

Many theories of autism have been proposed [28], implicating genetics (many genes have been linked to ASD), perinatal and environmental factors, stress, infections, vaccines, influence of various chemicals, etc. Genomic research projects related to autism run into hundreds of millions of dollars but their impact on translational research is small. These projects have been sponsored by the Autism Genome Project (AGP), an international autism genetics research consortium co-funded by Autism Speaks, the Medical Research Council, Canadian Institutes of Health Research, Health Research Board (Ireland), Genome Canada, the Hilibrand Foundation, and Autistica [21, 24]. However, none of the ASD theories has predictive power, all genetic correlations are 
quite weak and do not imply causality. One problem with the reliable diagnosis of ASD is the diversity and gradations in severity of symptoms, as well as changes in the brain of autistic people.

\subsection{Patophysiology}

An important question is whether there is a unifying mechanism at molecular, cellular or system level responsible for ASD? Autism may be a large set of disorders with diverse mechanisms, like intellectual disability that may be due to many reasons. Over 60 gene mutations have been implicated [12, 21], but they are linked to only about $20 \%$ of all the cases, with each single gene mutation correlated very weakly with autism. Other suggested reasons for the development of autism include:

- disorders caused by genetic mutations that converge on a few common molecular pathways alteration of brain development soon after conception, influenced by environmental factors,

- abnormal formation of synapses and dendritic spines due to the poorly regulated synthesis of synaptic protein, an excess of neurons that causes local overconnectivity,

- unbalanced excitatory-inhibitory networks.

There are many conditions comorbid to autism spectrum disorders:

- increased 10-20\% brain size in childhood, expansion of cerebral gray and white matter and cerebellum that disappears with age;

- distributed neocortical system disorder, but also various metabolic dysfunctions;

- mental retardation observed in $60-70 \%$ of cases;

- $40 \%$ autistic children have some form of epilepsy, $30 \%$ suffer from epileptic seizures;

- absence of spasticity or vision/hearing loss;

- lack of focal dysfunctions.

Moreover, there is common belief that patients with ASD symptoms are more frequently due to problems with association and prefrontal cortex rather than sensorymotor areas.

\subsection{Symptoms}

Many different symptoms of autism have been identified (see Fig. 1), some of them seem to be contradictory:

- difficulty in mixing with other children, social contacts,

- little or no eye contact,

- preference to be alone, aloof manner,

- inappropriate attachment to various objects,

- children may not want cuddling or act cuddly,

- spins objects, sustained odd and repetitive play and movements,

- inappropriate laughing and giggling, 

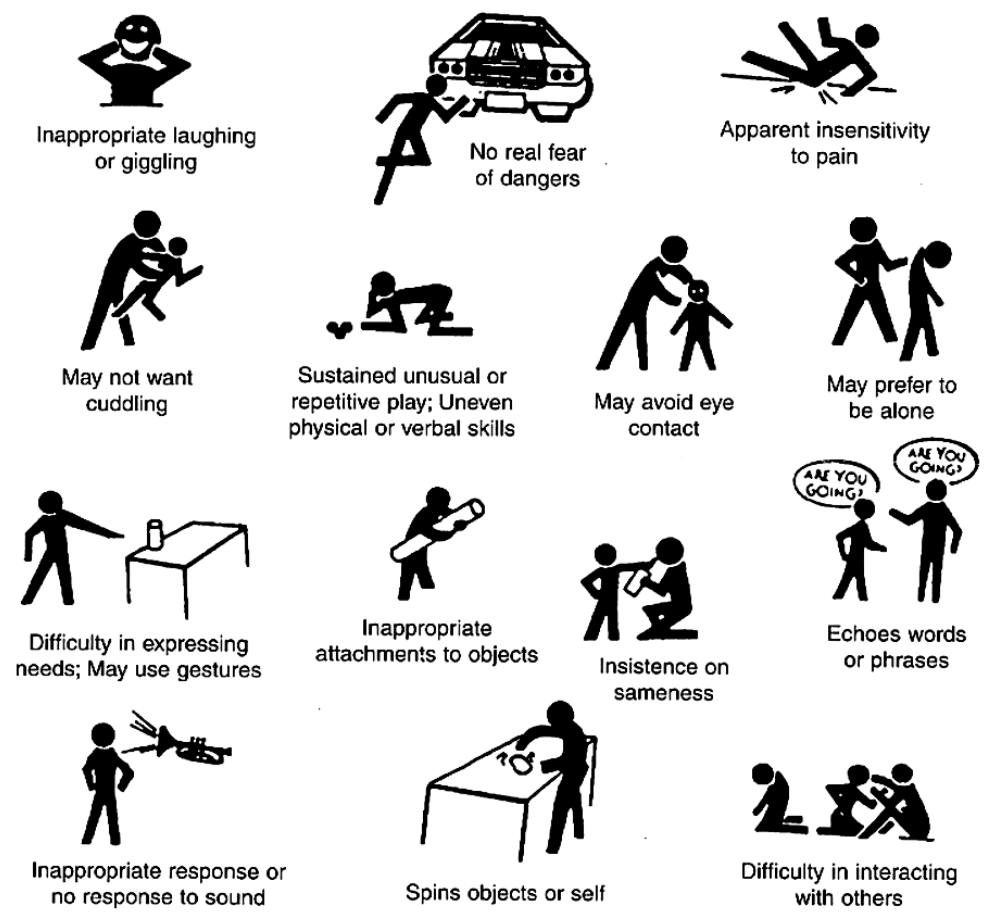

Figure 1. Selected symptoms of autism (chart from the Autism Society of America)

- insistence on sameness, resists changes in routine,

- noticeable physical overactivity, or extreme underactivity,

- tantrums, or extreme distress for no apparent reason,

- echolalia, repeating without apparent understanding words or phrases in place of normal language,

- apparent insensitivity to pain,

- no response to verbal cues, sometime act as deaf ignoring loud sounds,

- oversensitivity to low level sensory stimulation,

- difficulty in expressing needs, gestures/pointing instead of words.

\section{Theories}

The book edited by Zimmerman [28] has 20 chapters with different theories of autism spectrum disorders. Only a few of them are briefly explained below.

\subsection{Minicolumnopathy}

Minicolumnopathy is a theory proposed by Casanova [7] in ch. 16. It is based on observation that average minicolumnar width is $25.7 \mathrm{~mm}$ in autistic patients, in contrast 


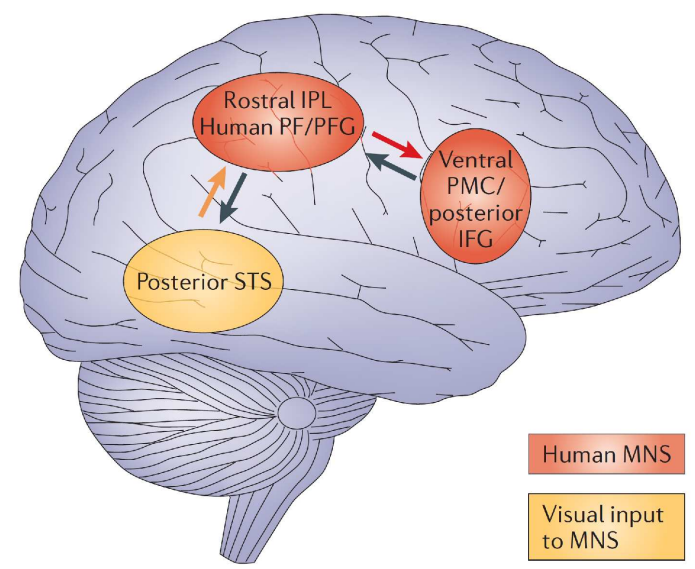

Figure 2. Brain areas involved in the mirror neuron subsystem [15]

to $27.2 \mathrm{~mm}$ in controls. This abnormality may cause smaller minicolumns in dorsolateral prefrontal cortex, increased cell density and shorter connecting fibers, which favor local computation.

Minicolumnar variability is the result of genetic and epigenetic influences that presumably reduce combinatorial diversity within overlapping networks, necessary for behavioral flexibility, leading to autistic symptoms. This theory is quite general and does not make any specific predictions.

\subsection{Mirror Neuron System}

According to the theory of Mirror Neuron System (MNS) [15] multimodal neurons in motor cortex react also to visual observations, helping to understand actions of others by simulating similar motor activity. Distortion in the development of the MNS interferes with the ability to imitate, leading to social impairment and communication difficulties, and may be responsible for the lack of the "theory of mind".

However there is also abnormal brain activation in many other circuits in ASD, not only in the mirror system. Moreover, the performance of children with ASD on various imitation tasks may be normal. Another large neural subsystem related to the representation of the self-structures, the default mode network [6], has also been implicated (see below). The impairment of these two systems may be the result of general underconnectivity between spatially separated brain areas [11].

\subsection{Underconnectivity theory}

According to the underconnectivity theory there is an excess of low-level (sensory) processes, with underfunctioning of high-level neural connections and synchronization $[11,19]$. fMRI and EEG studies suggest local overconnectivity in the cortex and weak 
functional connections to/from frontal lobes. Underconnectivity is observed mainly within each hemisphere of the cortex. Autism may be in this view a disorder of the association cortex. The theory does not explain how and why this underconnectivity can arise, and how does it explain many specific ASD symptoms.

"Default brain network" (cingulate cortex, mPFC, lateral PC) shows low activity for goal-related actions; it is active in social and emotional processing, mindwandering, daydreaming [6]. Activity of the default network is negatively correlated with the "action network" (conscious goal-directed thinking), but this is not the case in autism. Perhaps this is a manifestation of underconnectivity, and shows disturbance of self-referential thought, necessary for development of the theory of mind.

\subsection{Function connectivity theory}

This model has been developed in the last 20 years by Nancy J. Minshew [28] in ch. 18: treating autism as a widespread disorder of association cortex, leading to the development of connectivity, and only secondarily as a behavioral disorder. It also stresses deficits of the working memory, planning, inhibition, and other executive functions, with superiority of locally oriented and perceptual brain operations. Although basic tenets of this approach may be correct [11] it is too general to make any specific predictions or detailed explanations.

\subsection{Empathizing-systemizing theory}

Empathizing-systemizing theory is a newer version of the extreme male brain theory [1]: autism as an extreme case of the male brain. Those individuals in whom systemizing is better than empathizing (according to psychometrical tests) suffer from ASD. This theory does not explain much except for the

\subsection{Imbalanced Spectrally Timed Adaptive Resonance Theory}

The iSTART theory [13], based on Grossbergs Adaptive Resonance Theory, assumes a breakdown of interactions of cognitive, emotional, timing, and motor processes involving prefrontal and temporal cortex, amygdala, hippocampus, and cerebellum as the cause of autistic symptoms. Autistic people have vigilance fixed at such a high setting that their learn representations are very concrete, or hyperspecific. While this is an interesting and rather comprehensive attempt to build a theory that explains many symptoms of autism parameters such as vigilance are hard to connect to the molecular level and physical processes in the brain.

\section{Attention deficits in neural models}

Models of development concentrating on attention deficits should explain how neural synchronization influences brain development and manifests in anomalous behaviour. Neural models reflect cellular neural properties that depend on molecular biology and ultimately genomics, and predict brain dynamics that may be tested directly 
using animal models at the neurophysiological level, or using experimental psychology techniques predicting behavior by simulating cognitive functions and relating them to clinical observations. Large-scale computer simulations of semi-realistic neural networks should be used, eventually incorporating genomic and proteomic data, as well as population genetics data, the prediction of behavior as a function of lowerlevel neural properties should allow for the precise characterization of phenotypes and thus the diagnosis, a new understanding of the causal chains and in effect will enable translational research in crucial areas of neuropsychiatric research.

\subsection{A Neural model - simple mechanism}

Our hypothesis is focused on the key role played by the attention processes in the development of neural systems. Attention is a process resulting from the synchronization of groups of neurons, existing feedbacks, competition, inhibition and multiple constraint satisfaction it does not involve any special subnetworks in the brain.

Attention deficits have been well documented in autistic children. For example, Kawakubo et al. concluded [17]: "These results demonstrate electrophysiological abnormalities of disengagement during visuospatial attention in adults with autism which cannot be attributed to their IQs. [...] We suggest that adults with autism have deficits in attentional disengagement and the physiological substrates underlying deficits in autism and mental retardation are different". Landry and Brynson [18] wrote: "Children with autism had marked difficulty in disengaging attention. Indeed, on $20 \%$ of trials they remained fixated on the first of two competing stimuli for the entire 8-second trial duration". To shift attention neurons need to desynchronize and then synchronize again. In the language of dynamical systems this means that the trajectory of the system, describing neural activity has to leave one attractor basin and jump to another basin. However, neural dysfunctions may make this process difficult. One cause may be due to the damage of leak ion channels that slow down the process of spontaneous depolarization of neurons. In such cases neurons remain locked in the same activity for a long time and the brain state resist changes, leading to hyperspecific memories, problems with disengagement of attention, and a general lack of flexibility of changing brain states. Lack of frequent changes of brain states in the developmental process will lead to underconnectivity because Hebbian correlation learning mechanisms will not be able to increase the strength of distal connections in a normal way.

Attractor dynamics of two models implemented in the Emergent simulator [20] has been studied to verify this hypothesis.

The first example is based on a model of visual recognition (Fig. 4), and it involves:

- recognition of two objects presented in the visual field;

- information is first processed by the on-off cells in the retina and passed to the thalamic lateral geniculate nuclei (LGN);

- from the LGN it is passed to the V1 and larger receptive fields of V2; 


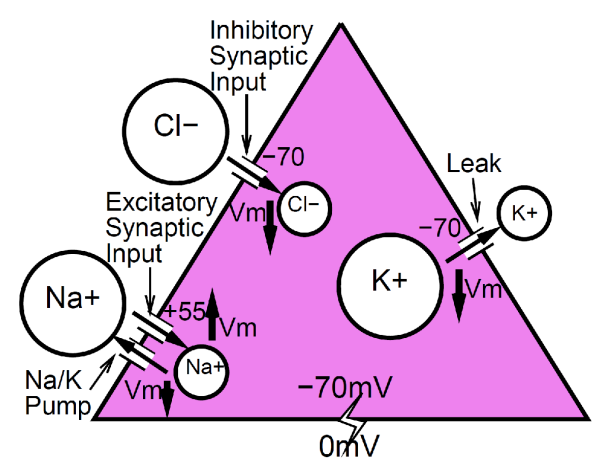

Figure 3. Model of artificial neuron implemented in the Emergent software [20] based on the simplified Hodgkin-Huxley model of neuron [14]

- the dorsal stream includes the V5/MT layers (Spat 1 and 2 in Fig. 4) that help to localize where the object is in the visual field and through the feedback connection helps to maintain the V2 and V1 activity focused on this object;

- the ventral stream includes V4/IT for object recognitions, and has connections with the V5/MT region.

Spat1 has recurrent activations and inhibition, focusing on a single object. In normal situations after a short time neurons desynchronize and synchronize on the second object, and as a result attention is shifted and the second object recognized. Damage to leak channels disables this process and the system cannot disengage attention from the first object for a long time. It is interesting that leak channels may also be damaged in the other direction letting large depolarizing current out, and thus making the system unstable, jumping from one object to the other. This is characteristic of the attention deficit hyperactivity disorder (ADHD).

Thus, relatively simple low-level problem with properties of neurons may lead to autism and ADHD. Considering the influence of such problems on the development a variety of symptoms may be explained.

However, realistic simulations of such processes require billions of neurons with trillions of connections. Such structures cannot be simulated without supercomputing. Even the simulation of our relatively simple model requires high computational powers. Each neural cell is being simulated according to the Hodgkin-Huxley model (Fig. 3) [14]. In the model each small part of the cell (so called compartment) is represented by its equivalent electrical circuit. The behavior of the circuit is described by a set of several nonlinear differential equations. The model becomes more complicated if we take into consideration the influence of other ionic channels, different kinds of synapses, and other parameters that may have influence on the behavior which is the point of our interest. The emergent simulation environment admits only relatively simple point neuron models. 

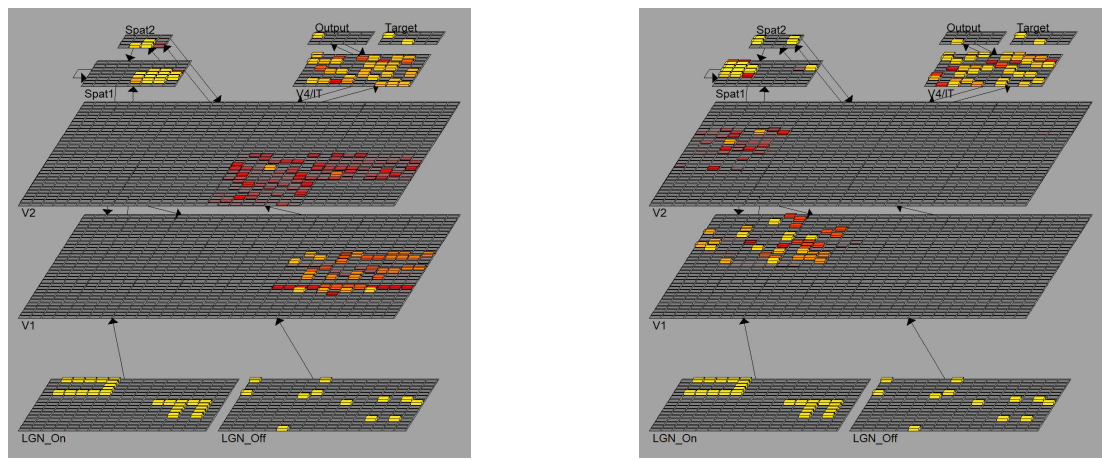

Figure 4. Model of visual recognition [20] based on [7]. Two steps of visual recognition simulation: on the left the first object was recognized, on the right after attention shifted to the second object

The model consisting of about $100 \mathrm{HH}$ cells with a relatively small number of connections (e.g., $30 \%$ of full connection) needs around 200 seconds to be simulated on an average desktop PC.

Increasing the number of simulated neurons in the model elongates the time of simulation $[26,25,27]$ because solving large numbers of differential equations present in $\mathrm{HH}$ model is very power consuming $[23,16]$. Simulating too small number of $\mathrm{HH}$ cells in the attention model may be not satisfactory as such ensembles have a strong tendency to synchronization. That is the main reason for increasing the number of cells in the particular fields in our planned experiments. Better biological correctness is also important at this stage. By moving to larger simulations we hope to observe phenomena with significantly better quality.

This is why more sophisticated simulations will be conducted in the parallel version of the GEneral NEural Simulation System (GENESIS) [5]. In addition some of the abovementioned parameters cannot be implemented directly into Emergent. Initial experiments have been already successfully completed on the local cluster, however, in future, for larger simulations we are going to benefit from the support of the Polish Grid Project (PL-Grid) [22]. We have some experience in conducting large $\mathrm{HH}$ simulations in CLUSTERIX project $[8,26]$.

\subsection{Attractors}

Attention results from the synchronization of neurons arising due to:

- inhibitory competition,

- bidirectional interactive processing,

- multiple constraint satisfaction.

Basins of attraction capture the dynamics of the system allowing for object recognition prompted by given input activations. In Fig. 5 the Fuzzy Symbolic Dynamics 
(FSD) visualization technique [9] is used to show attractors of a semantic layer with 140 units in a model of reading [20]. The trajectory moves around the phase space, jumping in a few steps from one basin attractor to the other, each corresponding to one of 40 learned concepts (words).
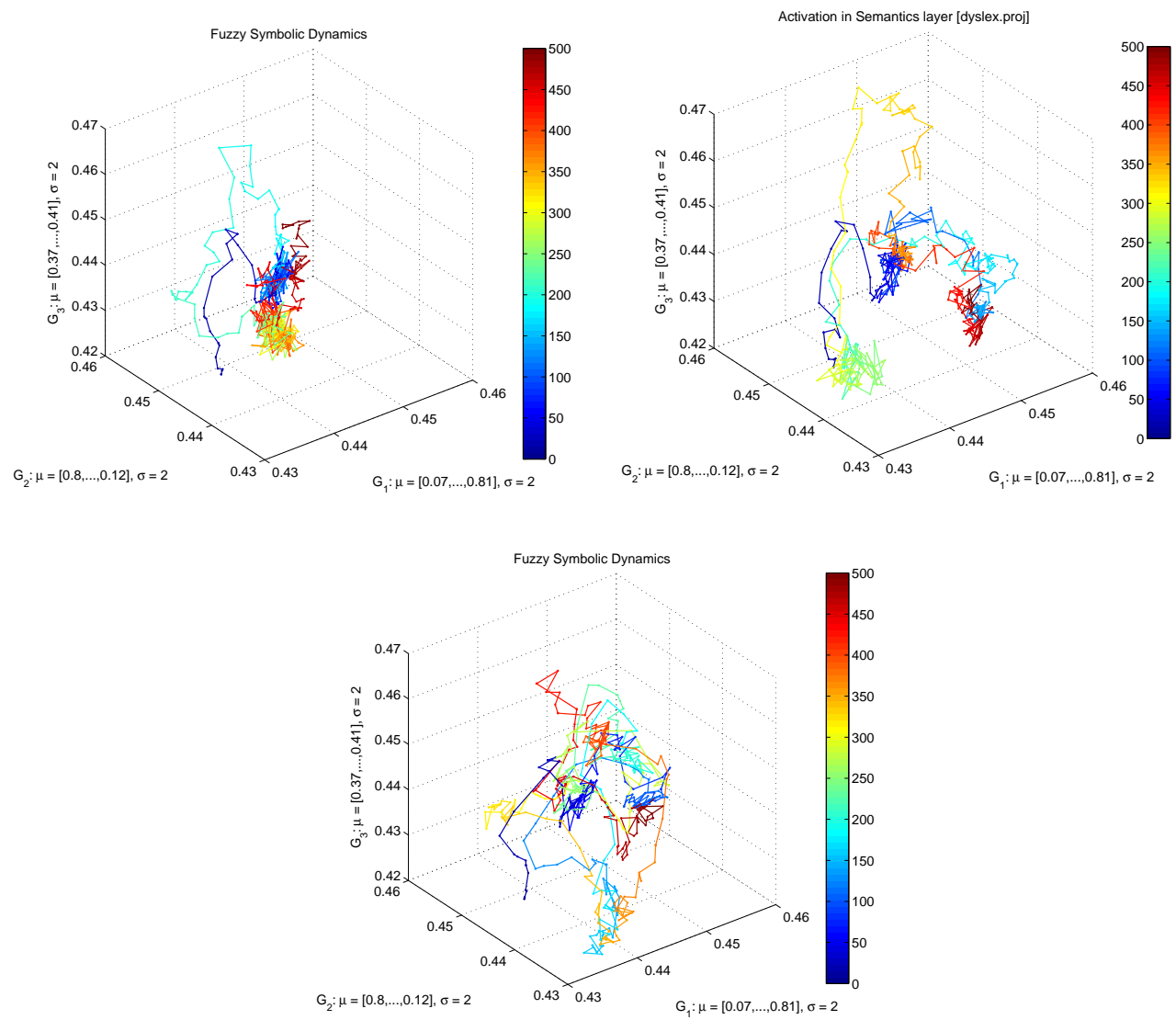

Figure 5. FSD visualization of attractors in a semantic layer with 140 units in weak (ASD, 3 basins), normal ( 5 basins), and strong (ADHD, 9 basins of attraction) accommodation cases (top, center and down respectively). Time flow (iterations) is represented by the color scale on the right

Results are shown for a weak (ASD), normal and strong (ADHD) neural accommodation case that results from a change of parameters regulating the activity of the leak channel (for more information about the accommodation mechanism see [20]). In the normal situation relatively large attraction basins lead to easy associations resulting in subsequent transitions from one basin to another, exploring the activation space. Weak accommodation prevents normal activation flow and in consequence leads to the formation of deep, strong, and tight attraction basins making it hard 
to move out of them (i.e. the system is focused on one stimuli, generalization and association is very weak). On the other hand, strong accommodation leads to the fast depolarization of neurons forming large, shallow attraction basins that prevent neural system from focusing long enough on one activity pattern, thus resulting in the inability to focus. More details about the experiment and the FSD technique itself can be found in [10].

\subsection{Consequences of Deep Attractor Basins}

Deep basins of attraction may lead to the following symptoms:

- deficits in attentional disengagement,

- overspecific memory for images, words, numbers, facts, movements,

- strong focus on a single stimulus, absorption, easy sensory overstimulation with weak signals,

- in motor cortex this can lead to repetitive movements due to the cyclic sequential attractors,

- generalization and associations should be quite poor;

- integration of different sensory modalities can be impaired and thus development of distal connections can proceed slowly;

- echolalia, repeating of words without understanding (no associations) may occur,

- fast changes of stimuli may be hard to noticed because stable attractor states cannot arise for sufficiently long time,

- faces can be ignored as they change so fast, so contact with caretakers can be difficult, gaze can be focused on simple stimuli;

- underconnectivity can lead to abnormal mirror neuron and default mode systems, impairing the normal development of social relations, the creation of a theory of mind, and emotional development.

This approach also explains why genetic research has found mutations that are so weakly correlated with ASD: in a given population of autistic patients only a small fraction will have a given mutation, many problems at the genetic/molecular level may influence neural depolarization and thus will show similar behavioral symptoms. Weak correlations will lead to problems for statistically-oriented research methods, as we cannot form relatively uniform groups of autistic people, each is going to be different. Results of many procedures will be inconclusive, for example effects of diet where several studies show some improvement, while other studies show no effect. Pharmacological and other treatments will have limited success. Until a better diagnostic at molecular/genetic level will help to stratify the individual cases development of efficient therapies will be unlikely.

\section{Concluding remarks}

Simple basic deficit with neural properties (leak channels) or connectivity can lead to a host of problems explaining various ASD symptoms. This disease will be very 
diverse, may be only parameterized in a continuous space because its severity and specific symptoms depend on so many parameters, extent and location of neural damage, developmental processes that lead to further dysfunctions of high-level systems, and numerous other factors.

Many insights from simple but general mechanisms may result. Our strategy is in agreement with the ideas of neuropsychiatric phenomics [4, 3], a new movement aimed at the systematic study of phenotypes. Neuropsychiatric symptoms and syndromes are investigated at the system level, relating them to cognitive systems and their functions, supported by neural systems. Properties of these systems result from cell biology, which in turn is related to molecular biology, linked to genetic and epigenetic processes. Correlations across the levels adjacent in this hierarchy are much stronger than between distant levels, giving a much better chance for understanding the causal factors and executing successful translational research projects.

The phenomics approach to neuropsychiatry requires expertise in genetics, molecular biology, cell biology, theoretical computational neuroscience, systems biology, behavioral sciences, as well as support from the information sciences, statistics, and mathematical modelling for data analysis, integration, visualization, and multilevel hypotheses testing. The creation of research groups with such broad expertise is a daunting task and may be achieved only through international collaboration on a wider scale.

Neural simulations are at the middle level, between molecular and behavioral levels. They give a chance for the understanding of the real reasons of causing behavior and linking network dynamics with molecular and genetic properties. Such simulations require high computational power. Their results will be presented during future workshops.

\section{Acknowledgements}

This research was supported by the Polish Ministry of Science and National Education (MNiSW/N519 5781 38).

\section{References}

[1] Baron-Cohen S.: Autism: The empathizing-systemizing (e-s) theory. Annals of the New York Academy of Sciences, 1156:68-80, 2009.

[2] Baron-Cohen S., Scott F., Allison C., Williams J., Bolton P., Matthews F., Brayne C.: Prevalence of autism-spectrum conditions. UK school-based population study The British Journal of Psychiatry, 194(6):500-509, 2009.

[3] Bilder R. M., Sabb F. W., Cannon T. D., London E. D., Jentsch J. D., Parker D. S., Poldrack R.A., Evans C., Freimer N.B.: Phenomics: the systematic study of phenotypes on a genome-wide scale. Neuroscience, 164(1), 2009. 
[4] Bilder R.M., Sabb F.W., Parker D.S., Kalar D., Chu W.W., Fox J., Freimer N.B., Poldrack R. A.: Cognitive ontologies for neuropsychiatric phenomics research. Cognitive Neuropsychiatry, 14(4-5):419-450, 2009.

[5] Bower J., Beeman D.: The Book of Genesis - Exploring Realistic Neural Models with GEneral NEural SImulation System. Springer, 1998.

[6] Broyd S.J., Demanuele C., Debener S., Helps S. K., James C. J., SonugaBarke E. J.: Default-mode brain dysfunction in mental disorders: A systematic review. Neuroscience \& biobehavioral reviews, 33:279-296, 2008.

[7] Casanova M.F.: The neuropathology of autism. Brain Pathology, 17:422-433, 2007.

[8] Clusterix.: Krajowy klaster linuksowy. http://www.clusterix.pcz.pl/, grudzień 2010.

[9] Dobosz K., Duch W.: Understanding neurodynamical systems via fuzzy symbolic dynamics. Neural Networks, 23(4):487-496, 2010.

[10] Duch W., Dobosz K.: Visualization for understanding of neurodynamical systems. Cognitive Neurodynamics, 5(2):145-160, 2011.

[11] Gepner B., Feron F.: Autism: A world changing too fast for a mis-wired brain? Neuroscience and Biobehavioral Reviews, 33(8):1227-1242, 2009.

[12] Geshwind D.H.: Autism: Many genes, common pathways? Cell, 135:391-395, 2008.

[13] Grossberg S., Seidman D.: Neural dynamics of autistic behaviors: cognitive, emotional, and timing substrates. Psychological Review, 113(3):483-525, 2006.

[14] Hodgkin A.L., Huxley A.F.: A quantitative description of membrane current and its application to conduction and excitation in nerve. Journal of Physiology, 117:500-544, 1952.

[15] Iacoboni M., Mazziotta J. C.: Mirror neuron system: basic findings and clinical applications. Ann Neurol, 62:213-218, 2007.

[16] Kamiński W.A.: Theoretical Neurocybernetics by Ryszard Tadeusiewicz, chapter Modelling of Single Neural Cells, pp. 75-86. Warsaw University Publishers, Warsaw, 2009.

[17] Kawakubo Y., Kasai K., Okazaki S., Hosokawa-Kakurai M., Watanabe K., Kuwabara H., Ishijima M., Yamasue H., Iwanami A., Kato N., Maekawa H.: Electrophysiological abnormalities of spatial attention in adults with autism during the gap overlap task. Clinical neurophysiology, 118(7):1464-1471, 2007.

[18] Landry L., Bryson S.: Impaired disengagement of attention in young children with autism. Journal of Child Psychology and Psychiatry, 45(6):1115-1122, 2004. 
[19] Müller R.A.: The study of autism as a distributed disorder. Mental Retardation and Developmental Disabilities Research Reviews, 13:85-95, 2007.

[20] O'Reilly R. C., Munakata Y.: Computational Explorations in Cognitive Neuroscience: Understanding the Mind by Simulating the Brain. MIT Press, Cambridge, Massachusetts, 2000.

[21] Pinto D., Pagnamenta A.T., Klei L., Anney R., Merico D., et al.: Functional impact of global rare copy number variation in autism spectrum disorders. Nature, 466(7304):368-372, 2010.

[22] PL-GRID.: The homepage of pl-grid project. http://www.plgrid.pl, December 2011.

[23] Tadeusiewicz R.: Theoretical Neurocybernetics. Warsaw University Publishers, Warsaw, 2009.

[24] Wallis F., Russell H.F., Muenke M.: Review: Genetics of attention deficit/hyperactivity disorder. Journal of Pediatric Psychology, 33(10):1085-1099, 2008 .

[25] Wojcik G. M.: Large simulations of mammalian visual system. In Science and Supercomputing in Europe, pp. 290-295. HPC-Europa Annual Project Directory, 2005.

[26] Wojcik G. M., Kaminski W. A.: Large scalable simulations of mammalian visual cortex. In Parallel Processing and Applied Mathematics, volume 3911 of Lecture Notes in Computer Science, pp. 399-405. Springer, 2005.

[27] Wojcik G. M., Kaminski W.A.: Grid-based simulations of mammalian visual system. In Proceedings of Cracow Grid Workshop 2005, pp. 384-389, 2006.

[28] Zimmerman A. W.: Autism: Current theories and evidence. Humana Press, Totowa, NJ, 2000.

\section{Affiliations}

Włodzisław Duch

Department of Informatics, Nicolaus Copernicus University, ul. Grudziądzka 5, 87-100, Torun, Poland, wduch@is.umk.p1

\section{Wiesław Nowak}

Institute of Physics, Nicolaus Copernicus University, ul. Grudziądzka 5, 87-100, Toruń, Poland, wiesiek@fizyka.umk.pl

\section{Jarosław Meller}

Department of Informatics, Nicolaus Copernicus University, ul. Grudziądzka 5, 87-100, Toruń, Poland, jmeller@chmcc.org

\section{Grzegorz Osiński}

Department of Informatics, Nicolaus Copernicus University, ul. Grudziądzka 5, 87-100, Toruń, Poland, gos@fizyka.umk.pl

\section{Krzysztof Dobosz}

Department of Informatics, Nicolaus Copernicus University, ul. Grudziądzka 5, 87-100, Torun, Poland, kdobosz@mat.umk.pl 


\section{Dariusz Mikołajewski}

Department of Informatics, Nicolaus Copernicus University, ul. Grudziądzka 5, 87-100, Toruń, Poland, darek.mikolajewski@wp.pl

\section{Grzegorz M. Wójcik}

Institute of Computer Science, Maria Curie-Sklodowska University, Pl. Marii

Curie-Skłodowskiej 1, 20-031, Lublin, Poland, gmwojcik@gmail.com

Received: 16.12 .2011

Revised: 17.04 .2012

Accepted: 23.04 .2012 